\title{
QUARKONIUM DECAY INTO HEAVY QUARK FLAVOURS
}

\author{
H. Fritzsch \\ University of Wuppertal
and \\ CERN -- Geneva \\ and \\ K. -H. Streng *) \\ University of Thppertal
}

\section{$A B S T R A C T$}

It is suggested that the production rate of heavy flavours (charm, $\psi(J)$, etc.) in the decay of heavy quarkonium states [e.g. T $9.4 \Sigma$ can be estimated using QCD perturbation theory. Especially we discuss the energy aistribution of $\psi(J)$ mesons produced in quarkonium decay.

*) Supported by Deutsche Forschungsgemeinschaft.

Ref.TH. 2499-CERN

5 May 1978 
1. INTRODUCTION

Recently several authors have proposed to abstract certain aspects of the hadronic final state in heavy quarkonium decay from QCD perturbation theory. Especially it was emphasized that it may be possible to obtain information about gluons and about the fragmentation of gluons into hadrons by looking at heavy quarkonium decay, e.g. by observing a three jet structure of the hadronic final state in the decay of $1^{--}$quarkonium resonances ${ }^{1)}$.

In this note we should like to continue this discussion by considering another application of the same idea, namely the decay of heavy quarkonia into other lower lying quarkonium states and into heavy flavors plus any number of normal hadrons, e.g. the decay of $T(9.4)^{2}$ into $\Psi(J)$ plus anything. (We interpret the $T(9.4)$ state as a bound state of a heavy quark $h$ and its antiquark. The electric charge of the $h$-quark is kept as a free parameter). We expect that a careful experimental study of these decays (rates, decay distributions etc.)will provide useful information about the dynamics of the strong interaction.

In our analysis we are encouraged also by the apparent success of simple ideas based on QCD perturbation theory for describing the production of $\Psi(J)$ and $T(9.4)$ in hadronic collisions. These processes are described by the fusion of two gluons ${ }^{3)}$ to give a heavy quarkonium state (dominant in nucleon-nucleon scattering) and by the annihilation of quarks and antiquarks ${ }^{4}$ ) into glue (dominant in nucleon-antinucleon scattering).

\section{HEAVY FLAVORS IN QUARKONIUM DECAY}

It is supposed that a heavy quarkonium state $\left(\mathrm{J}^{\mathrm{PC}}=1^{--}\right)$ decays into three "massless" gluons which materialize by fragmenting into hadrons. Although it is unknown how this materialization proceeds in detail, it seems reasonable to assume that gluons tend to fragment into hadrons composed of the light quark flavors, e.g. pions, p-mesons, etc. We do not expect gluons to have any tendency to fragment into hadrons composed of heavy quarks, e.g. charm. Within the QCD approach we expect that heavy flavors are produced in heavy quarkonium decay only by a process in which a virtual timelike gluon of large invariant mass is produced in the initial decay process and subsequently decays into a pair of heavy flavors. 
This process is described in Fig. (1). Although the production of a specific state will depend on unknown details of the strong interactions, the total rate for producing heavy flavors can be estimated in a reliable way as follows. It is well-known that in $e^{+} e^{-}$-annihilation the cross-section for producing a specific flavor is approximately equal to the cross-section for producing a pair of free quarks, if one averages over the various resonance bumps (see eg. ref. (5)). We apply the same idea to gluons and suppose that we can estimate the total rate for producing a pair of heavy flavors in quarkonium decay by calculating the rate for producing a pair of free heavy quarks. Similar ideas have been used to estimate the production rate for heavy flavors in hadronic scattering (see eg. ref. (4)). As an example we consider the decay $T \rightarrow$ charm + anticharm + anything.

One finds

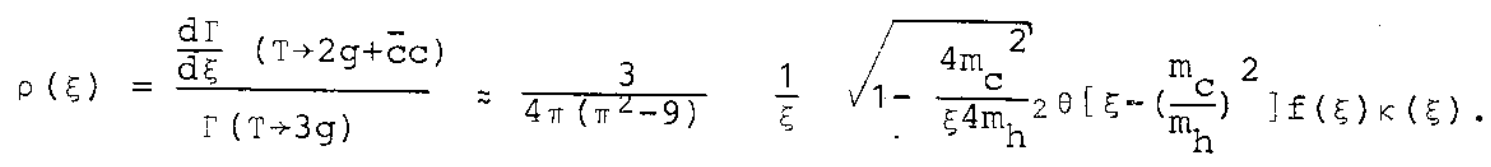

Here $g$ denotes a color octet gluon. The dimensionless parameter $\xi$ is defined as $\mathrm{q}^{2} / 4 \mathrm{~m}_{h}^{2}$, where $\mathrm{q}^{2}$ is the virtual gluon mass (mass of $c \bar{c}$ pair). The function $f(\xi)$ describes the decay of $T$ into two massless and one massive gluon and is given by $\mathrm{FN}$ )

$$
\begin{aligned}
& f(\xi)=\int \mathrm{dE}_{1} \mathrm{dE}_{2} \mathrm{dE_{3 }} F\left(\xi, \mathrm{E}_{1}, \mathrm{E}_{2}, \mathrm{E}_{3}\right) \delta\left(2 \mathrm{~m}_{\mathrm{h}}-\mathrm{E}_{1}-\mathrm{E}_{2}-\mathrm{E}_{3}\right) \\
& \left(\mathrm{E}_{1} \text { : energy of massive gluon, } \mathrm{E}_{2}, \mathrm{E}_{3}=2 \mathrm{~m}_{\mathrm{h}}-\mathrm{E}_{1}-\mathrm{E}_{2}\right. \text { : energies of } \\
& \text { massiess gluons), }
\end{aligned}
$$

where $F$ is defined by

$$
\begin{aligned}
F\left(\xi, E_{i}\right) & =F_{1}+\frac{1}{2 m_{h}\left(E_{1}-\xi\right)} F_{2}+\frac{1}{4 m_{h}{ }^{2}\left(E_{1}-\xi\right)^{2}} F_{3}+2 m_{h} \xi \cdot F_{4}+4 m_{h}{ }^{2} \xi_{5} F_{5} \\
F_{1} & =\frac{1}{E_{2}{ }^{2} E_{3}{ }^{2}}\left(E_{2}{ }^{2}+E_{3}{ }^{2}+\frac{3}{2} m_{h}{ }^{2}+\frac{3}{8} E_{1}{ }^{2}-m_{h} E_{1}\right) \\
F_{2} & =-1+m_{h}\left(E_{1}-4 m_{h}\right)\left(\frac{1}{E_{2}^{2}}+\frac{1}{E_{3}^{2}}\right) \\
& +\frac{1}{E_{2} E_{3}}\left(8 m_{h}{ }^{2}+\frac{1}{2} E_{1}{ }^{2}-4 m_{h} E_{1}-3 m_{h}{ }^{2} E_{1}\left(\frac{1}{E_{2}}+\frac{1}{E_{3}}\right)\right)
\end{aligned}
$$




$$
\begin{aligned}
\mathrm{F}_{3} & =8 \mathrm{~m}_{\mathrm{h}}^{2}+2 \mathrm{~m}_{\mathrm{h}} \mathrm{E}_{1}+\mathrm{m}_{\mathrm{h}}\left(\mathrm{E}_{1}{ }^{2}-3 \mathrm{~m}_{\mathrm{h}} \mathrm{E}_{1}-14 \mathrm{~m}_{\mathrm{h}}{ }^{2}\right)\left(\frac{1}{\mathrm{E}_{2}}+\frac{1}{\mathrm{E}_{3}}\right) \\
& +\mathrm{m}_{\mathrm{h}}^{2}\left(4 \mathrm{~m}_{\mathrm{h}}^{2}+\frac{1}{2} \mathrm{E}_{1}{ }^{2}\right)\left(\frac{1}{\mathrm{E}_{2}{ }^{2}}+\frac{1}{\mathrm{E}_{3}{ }^{2}}\right)+\frac{12 \mathrm{~m}_{\mathrm{h}}}{\mathrm{E}_{2} \mathrm{E}_{3}} \\
\mathrm{~F}_{4} & =\frac{1}{\mathrm{E}_{2}{ }^{2} \mathrm{E}_{3}{ }^{2}}\left(\frac{3}{4}+\frac{1}{8} \frac{\mathrm{E}_{2} \mathrm{E}_{3}}{\mathrm{~m}_{\mathrm{h}}{ }^{2}}-\frac{3}{8} \frac{\mathrm{E}_{1}}{\mathrm{~m}_{\mathrm{h}}}\right) \\
\mathrm{F}_{5} & =\frac{1}{32 \mathrm{~m}_{\mathrm{h}}{ }^{2} \mathrm{E}_{2}{ }^{2} \mathrm{E}_{3}}{ }^{2} .
\end{aligned}
$$

In the limit $\xi \rightarrow 0$ (i.e. $q^{2} \rightarrow 0$ ) the function $F$ approaches the function describing the energy distribution of the three photons in orthopositronium decay.

$$
F\left(O, E_{1}, E_{2}, E_{3}\right)=\left(\frac{m_{h}-E_{1}}{E_{2} E_{3}}\right)^{2}+\left(\frac{m_{h}-E_{2}}{E_{1} E_{3}}\right)^{2}+\left(\frac{m_{h}-E_{3}}{E_{1} E_{2}}\right)^{2} .
$$

The function $f(\xi)$ is displayed in Fig. (2). For our further consideration we neglect the $q^{2}$-dependence of $k$ in eq. (1) and use $k=1 / 3$ throughout the whole relevant $q^{2}$-range. The function $\rho(\xi)$ is displayed in Fig. (3). One observes that the maximum of decay rate is obtained for $\xi=0.15$, i.e. $\mathrm{q}^{2}=3.6 \mathrm{GeV}^{2}$. Furthermore we find, using eq. (1):

$$
\frac{\Gamma(T \rightarrow \bar{c} c+x)}{\Gamma(\Upsilon \rightarrow \text { hadrons })} \approx \int_{\frac{\left(2 m_{c}\right)^{2}}{\left(2 m_{h}\right)^{2}} d \xi_{\rho}(\xi) \approx 0.11 \cdot \kappa,}^{1}
$$

which is 3.8 for $k=1 / 3$.

The average energy of the two gluons ("gluon jets") emitted in the decay is $1.84 \mathrm{GeV}$, the average energy of the charmed quarks 
is $2.9 \mathrm{GeV}$. The average invariant mass of the charmed pair is $5.25 \mathrm{GeV}$.

\section{CHARM PRODUCTION IN T-DECAY}

Charmed particles will be produced in $T$-decay if $q^{2}>\left(2 M_{D}\right)^{2}=(3.72 \mathrm{GeV})^{2}$. Note that the $\bar{c}$-system produced by the disintegration of the massive gluon is in a color octet configuration. In order to evolve as a physical charm-anticharm system, the $\bar{c} c-s t a t e$ has to emit a color octet gluon, which in principle can be arbitrarily soft. We suppose (see also ref. (4)) that this color rearrangement occurs with probability unity and does not perturb our numerical calculation given above.

The production rate for charmed particles is obtained by carrying out the integral

$$
\frac{\Gamma(T \rightarrow \text { charm }+ \text { anticharm }+x)}{\Gamma(T \rightarrow \text { hadrons })}=\int_{\frac{\left(2 M_{D}\right)^{2}}{M_{T}^{2}}}^{1} \rho(\xi) d \xi
$$

which is 2.7 for $k=1 / 3$.

The best (and probably the only) way to see the production of charmed particles in $T$-decay is by looking for their leptonic decay modes. Taking a $10 \%$ leptonic branching ratio for the decay of charmed particles, the rate for producing e.g. a single muon (and its invisible neutrino) is given by

$$
\Gamma(\tau \rightarrow \mu+x) / \Gamma(T \rightarrow \text { hadrons }) \approx 0.5 \% \text {. }
$$

The average energy of the charmed quarks produced in the $T$-decay is about $2.9 \mathrm{GeV}$, i.e. the muons emitted in the subsequent weak decay of the charmed particles should have energies of the order of $0.5-1 \mathrm{GeV}$.

\section{4. $\Psi(\mathrm{J})$ PRODUCTION IN T-DECAY}

If the virtual gluon has an invariant mass which is less than $\left(2 M_{D}\right)^{2}$, the $\bar{c} c$ pair will manifest itself by the production of a $\bar{c} c-$ meson. The probability to produce a particular state, e.g. $\Psi(\mathrm{J})$, depends on dynamical details of the strong interactions (in 
particular on how the color octet $\bar{c}$ configuration arranges itself to evolve as a color singlet charmonium state by emitting soft gluons)and cannot be calculated in a reliable way. The simplest possibility to arrange this is to emit just one soft gluon in which case a $C=+1$ charmonium state ( $x$-state, $n_{C}$ ) is produced. Thus one may expect in particular that a sizable fraction of $\psi(\mathrm{J})$ production in $\mathrm{T}$-decay proceeds via the initial production of a $x$-state which cascades down to $\psi(\mathrm{J})$ by a radiative decay.

The situation here is completely analogous to the production of $\psi(J)$ in hadronic reactions (see e.g.ref. $(4,5)$ ). Using eq. (5) and eq. (6) we estimate the total prnduction rate for charmonium states in $\mathrm{r}$-decay:

$$
\frac{\Gamma(T \rightarrow \text { charmonium })}{\Gamma(T \rightarrow \text { hadrons })} \approx 1.1 \%
$$

A similar calculation performed in order to obtain the $\psi(\mathrm{J})$ production rate in hadronic reactions ${ }^{4,5}$ ) shows that the total $\psi(\mathrm{J})$ production rate is of the same order of magnitude than the total charmonium production rate. Thus we conclude

$$
\frac{\Gamma(T \rightarrow \psi(J)+x)}{\Gamma(T \rightarrow \text { hadrons })} \sim 18
$$

Since the production of $\psi(J)$ can easily be observed by detecting the $\mu^{+} \mu^{-}$pairs, we expect that $\psi(\mathrm{J})$ production can be measured in $\mathrm{T}$-decay.

It is interesting to consider the energy spectrum of the $\psi(J)$ mesons in $T$-decay, which will be essential identical to the spectrum of virtual massive gluons of mass $~ 3.1-3.5 \mathrm{GeV}$. In Fig. (4) we display the momentum distribution function (unnormalized) $F(n)\left[n=\frac{p[\psi(J)]}{p[\psi(J), \max .]}\right.$ for $q^{2}=[3.1 \mathrm{GeV}]^{2}$, i.e. $p[\psi(J), \max ]=.4.2 \mathrm{GeV}$. The maximum momentum for the $\psi(J)$ mesons is obtained for the configuration where two gluons are emitted collinear and opposite to the direction of the $\psi(\mathrm{J})$ momentum.

Fig. (4) is perhaps the most interesting result of this paper; the distribution of the $\psi(J)$ mesons in $T$-decay provides an interesting test of $\mathrm{QCD}$ and the three gluon decay hypothesis for 
T-decay. Note that the probability distribution reaches its maximum for the configuration with the maximum of momentum $(4.2 \mathrm{GeV} / \mathrm{c})$. Such events will consist of a $\psi(J)$ meson emitted in one direction, eventually accompanied by a photon, and a jet of hadrons emitted in the opposite direction.

\section{CHARMONIUM PRODUCTION VIA P-WAVE T-DECAY}

The $\mathrm{O}^{+}$and $2^{+}$P-wave $\bar{h}$-states belonging to the $\mathrm{T}$-family are expected to decay hadronically via the emission of two gluons. Following the same line of argument as presented above we expect that about $1 \%$ of the hadronic decays of the P-wave states to contain a $\psi(J)$ meson. Such events are expected to look rather distinct. They will consist of two jets, one gluon jet carrying a large momentum, and a $\psi(\mathrm{J})$ meson carrying a large momentum into the opposite direction. If we assume a mass of $9.7 \mathrm{GeV}$ for the $\mathrm{C}_{n}=+1$ P-wave states, the momentum of the gluon jet will be about $4.4 \mathrm{GeV} / \mathrm{c}$. (see Fig. (5)).

Note that often the $\psi(J)$ will be accompanied by a photon, originating from the radiative decay of a p-wave charmonium state. If the massive gluon is massive enough to produce charm-anticharm states, it will do so. The result will be a pair of charmed mesons emitted with high momentum in the direction opposite to the gluon jet.

\section{CONCLUSION}

In this paper we have emphasized that it will be useful to study the production of charm and charmonium states in the decay of heavy quarkonium states. Such a study will provide interesting informations about $\mathrm{QCD}$ and the role played by gluons in the decay of heavy quarkonium states. Especially the energy distribution of $\psi(J)$ mesons produced in heavy quarkonium decay (see Fig. (4)) constitutes an interesting test.

\section{ACKNOWLEDGMENT}

One of us (H.F.) is indebted to G.Parisi for a useful conversation about some of the ideas discussed in this paper. 
FOOTNOTE

In the derivation of eq. (1) we have neglected terms proportional to $\left(\vec{p}_{c}^{2} / q^{2}\right)$, where $\vec{p}_{C}$ denotes the three-momentum of the $c$ or $\bar{c}$ quark. Since the production cross-section is dominated by contributions from the kinematical region where the c quarks move nonrelativistically in the rest system of the virtual gluon, this is good approximation.

\section{FIGURE CAPTIONS}

Fig.(1): Heavy quarkonium decay into a pair of heavy flavors plus normal hadrons.

Fig. (2): The function $f(\xi)$ defined in eq. (1).

Fig. (3): The branching ratio eq. (1) as a function of $\xi=q^{2} /\left(2 m_{h}\right)^{2}$.

Fig. (4): The momentum distribution of $\psi(\mathrm{J})$ mesons in $T$-decay.

Fig. (5): The decay of a $C_{n}=+1 \bar{h} h-s t a t e$ into a gluon jet and a massive gluon producing a $\psi(J)$-state. 


\section{REFERENCES}

1.) T.A.Grand, Y.J.Ng and S.H.Tye,

Phys.Rev. D16, 3251 (1977).

K. Koller and T.F. Walsh,

Phys.Lett. 72B, (1977) 227,

S.Brodsky, D.G.Coyne, T.A.Grand and R.R.Horgan,

SLAC-PUB-2047 (1977),

H.Fritzsch and K.H.Streng,

Phys.Lett.74B, 90 (78).

A.De Rujula, J.Ellis, E.G.Floratos and M.K.Gaillard, CERN preprint TH 2455 (1978).

2.) S.W.Herb et al., Phys.Rev.Lett. 39 (1977) 252.

3.) M.B.Einhorn and S.D.Ellis,

Phys.Rev. 12 (1975) 2007.

M.Glück, J.F.Owens and E.Reya,

Florida State University preprint FSU HEP 77-08-10 (1977).

4.) H.Fritzsch, Phys. Lett 67B (1977) 217.

5.) H.Fritzsch and H.Leutwyler, Phys.Rev. D10 (1974) 1624 .

E.Poggio, H.Quinn, and S.Weinberg,

Phys.Rev. D13 (1976) 1958. 


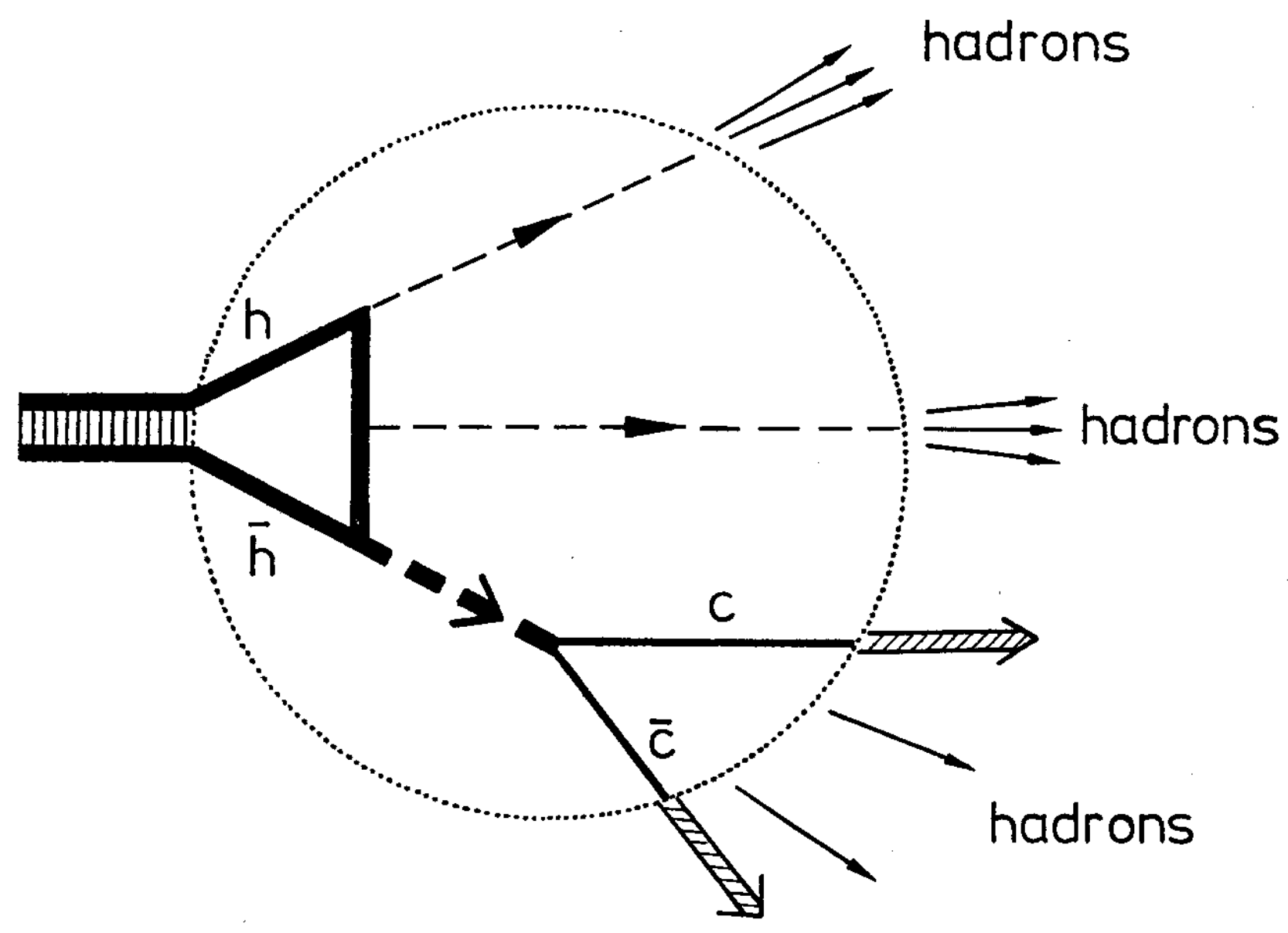

Fig. 1 


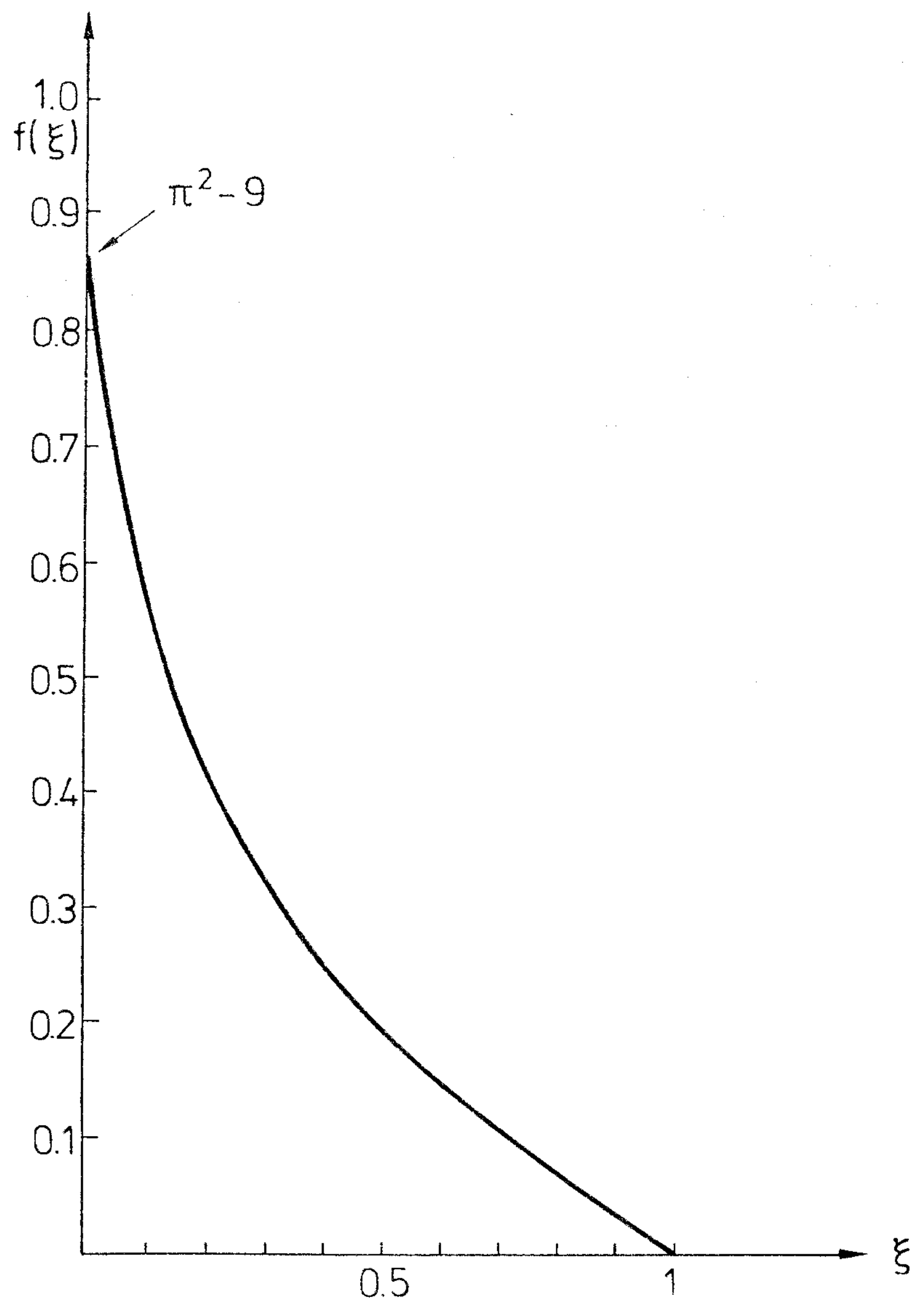

Fig. 2 


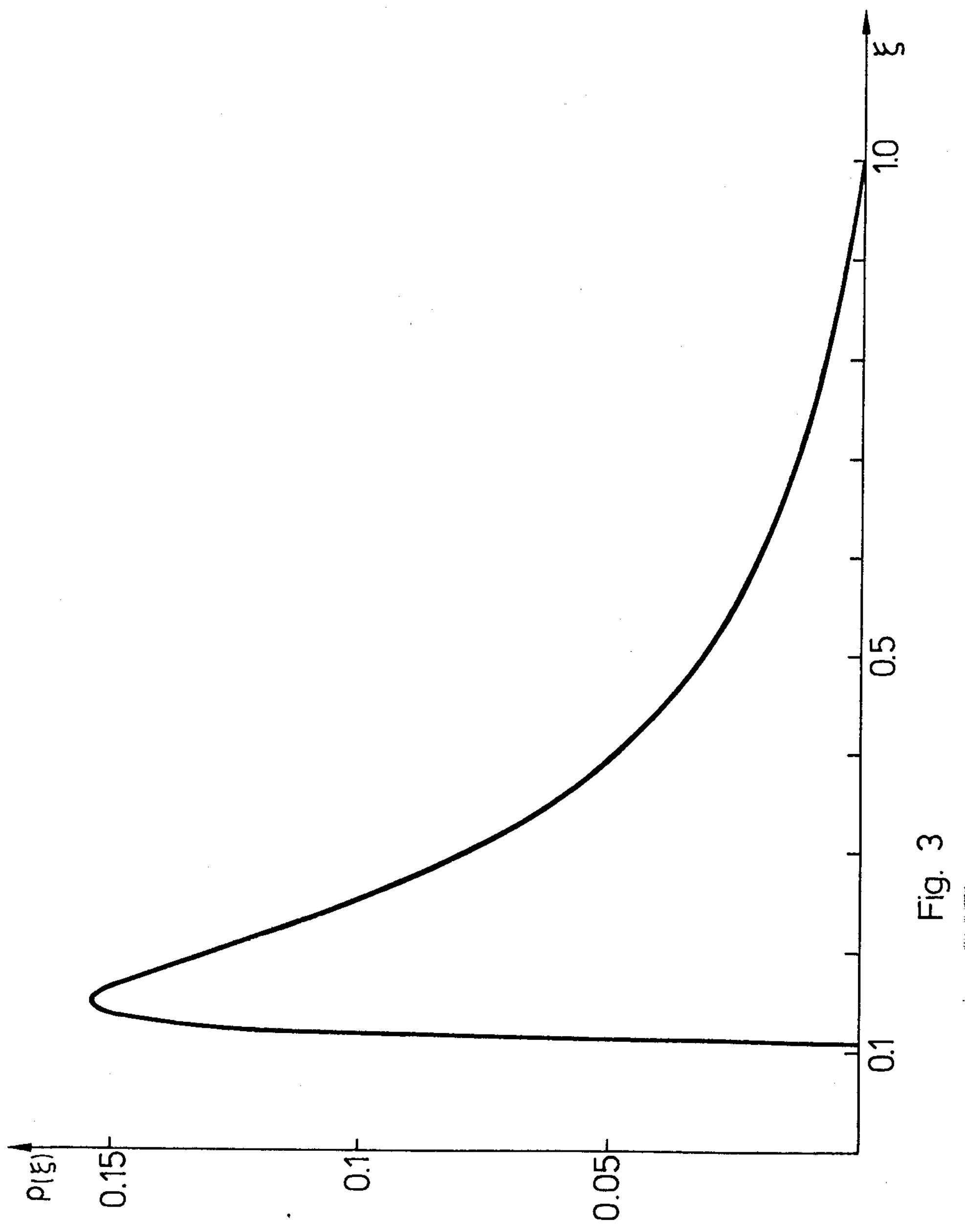




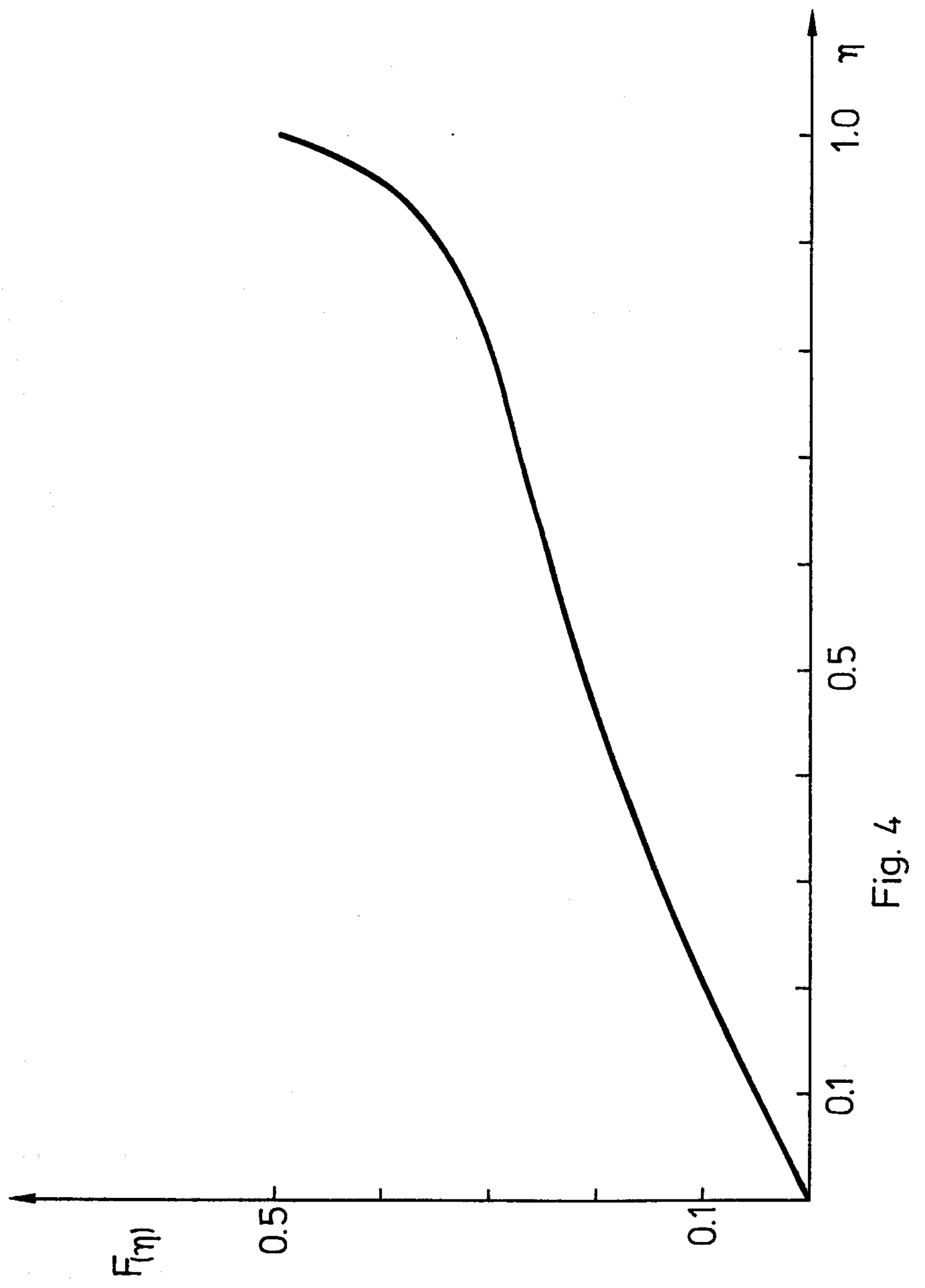




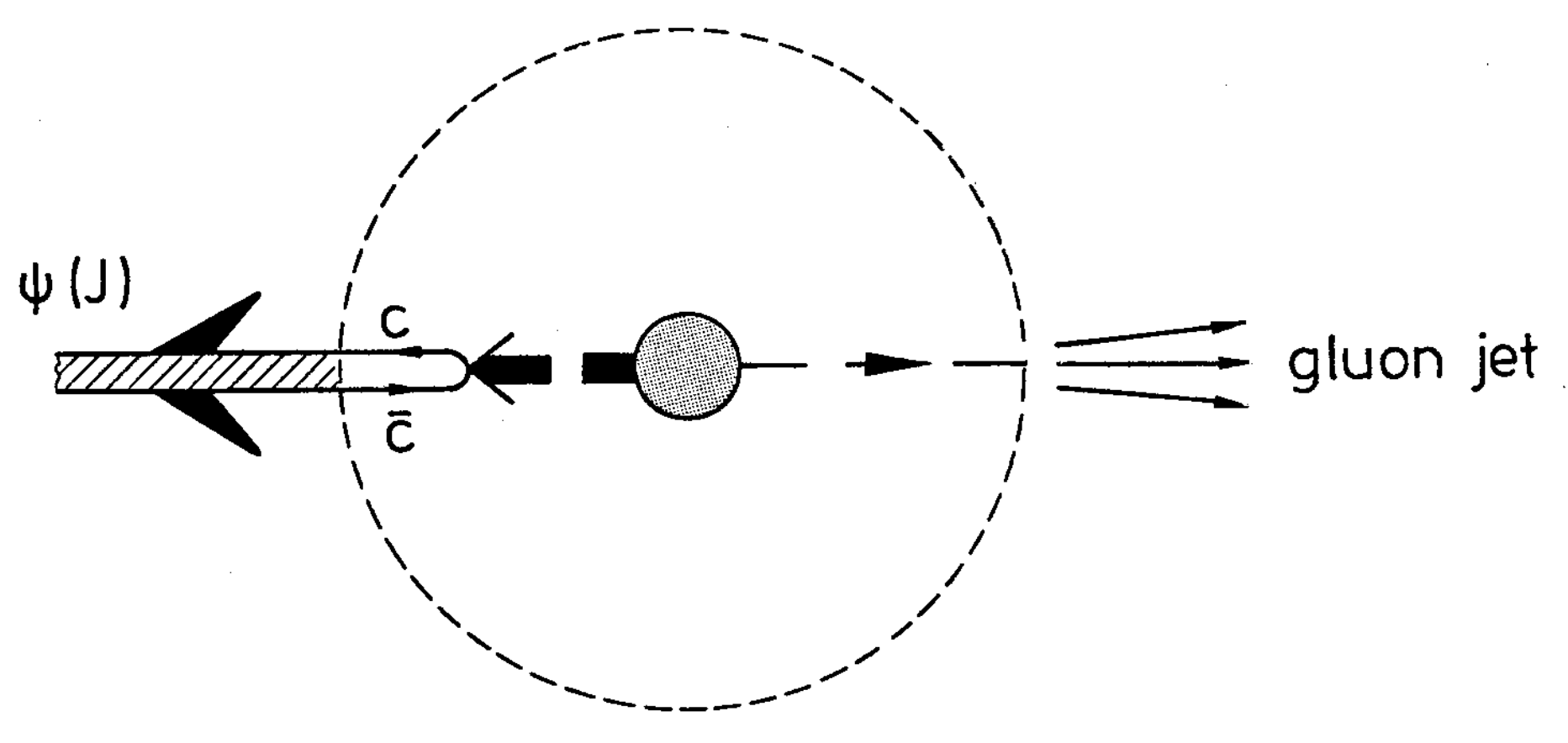

Fig. 5 